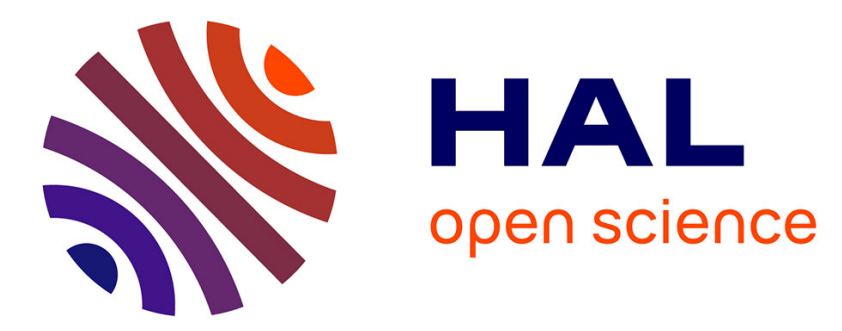

\title{
Using Fractional Calculus for Cooperative Car-Following Control
}

\author{
Carlos Flores, Vicente Milanés, Fawzi Nashashibi
}

\section{To cite this version:}

Carlos Flores, Vicente Milanés, Fawzi Nashashibi. Using Fractional Calculus for Cooperative CarFollowing Control. Intelligent Transportation Systems Conference 2016, IEEE, Nov 2016, Rio de Janeiro, Brazil. hal-01382821

\section{HAL Id: hal-01382821 \\ https://hal.inria.fr/hal-01382821}

Submitted on 17 Oct 2016

HAL is a multi-disciplinary open access archive for the deposit and dissemination of scientific research documents, whether they are published or not. The documents may come from teaching and research institutions in France or abroad, or from public or private research centers.
L'archive ouverte pluridisciplinaire $\mathbf{H A L}$, est destinée au dépôt et à la diffusion de documents scientifiques de niveau recherche, publiés ou non, émanant des établissements d'enseignement et de recherche français ou étrangers, des laboratoires publics ou privés. 


\title{
Using Fractional Calculus for Cooperative Car-Following Control
}

\author{
Carlos Flores, Vicente Milanés, Fawzi Nashashibi
}

\begin{abstract}
The Cooperative Adaptive Cruise Control (CACC) is one of the most promising aiding systems to improve traffic flow in highways. When it comes to design a proper control algorithm, robustness against non-modeled dynamics and noise plays a key role not only for improving controller performance but also for increasing the ability of handling heterogeneous vehicle strings. This paper proposes a fractional order controller that is able to deal with non-modeled dynamics whereas keeping simplicity and a low computational cost. System robustness and string stability responses are analyzed for a string of six vehicles, showing a good performance.
\end{abstract}

\section{INTRODUCTION}

Traffic flow capacity in urban and peri-urban areas have significantly increased in recent years. The increment in the number of vehicles together with the infrastructure limitations are causing multiple traffic jams, affecting drivers' daily life ${ }^{1}$. Several solutions to this problem have been proposed, such as the employment of Vehicle Ad Hoc Networks [1] to propagate traffic information among the vehicles and also traffic prediction algorithms with In-Car advice to the driver [2]. Both systems are mainly related to infrastructure adaptation by controlling traffic flow, being able to update intelligent traffic panels for a dynamic maximum vehicle speed in each road depending on the traffic flow.

From the vehicle control perspective, automated speed control systems are a suitable solution to deal with these traffic flow problems. For instance, cruise control system (CC) is a commercial feature whose main purpose is maintaining the vehicle speed in a more stable way than a common driver would. However, it losses its benefits in urban environments where continuous speed changes occur. Therefore, an improved version of the $\mathrm{CC}$, the adaptive cruise control (ACC), proposes an approach to car-following systems which adapts the ego vehicle speed, maintaining a fixed headway time gap with the preceding car by measuring the relative distance between the two vehicles using a front range sensor.

In the recent years, the inclusion of the V2V and V2I communications in the transportation sector has allowed multiple applications to enhance the traffic and road safety [3]. For example, an improved version of the ACC system, the Cooperative Adaptive Cruise Control (CACC), has demonstrated that by adding communication links between ACC-equipped vehicles, shorter inter-distances can be achieved. This yields an increase in traffic throughput [4], and if the distances are short enough, the aerodynamic drag is significantly reduced (specially for heavy duty trucks [5]).

\footnotetext{
${ }^{1}$ http://ec.europa.eu/transport/road_safety/specialist/statistics/index_en.htm
}

Different CACC implementations have been carried out in recent years. Most of the proposed approaches have been focused on different type of integer-order robust controllers [6]; but other techniques as MPC [7] or even fuzzy logic [8] have been proposed to provide stable CACC algorithms.

Focusing on robust controllers, it can be found that up to $95 \%$ of current control systems are based on PID controllers [9], providing robustness whereas keeping the tuning simplicity. Their main limitations arise by the fact that the controller design assumes integer order models that might be far from the real system response. To cope with this limitation, fractional order calculus has surged recently as a powerful control technique to deal with some of the limitations of PID controllers, whereas keeping the simplicity in terms of computational cost. Some recent examples for fractional order adaptive cruise control applications outperforming classical approaches, can be found in [10], [11].

This article presents a fractional order controller approach that allows a ACC-equipped vehicle, with V2V communication, to smoothly maintain the distance gap respect to the preceding vehicle. It will also be evaluated the performance of the proposed controller when implemented in a string of CACC-equipped vehicles.

The rest of this work is presented as follows. Section II reviews the mathematical tool fractional order calculus, its theory and applications. A description of the problem, including the spacing policy and the control structure for the CACC car-following is carried out in section III. Next, the controller design procedure is detailed in section IV. Later, the results for the developed controller with the string stability study are presented in section $\mathrm{V}$; finally some concluding remarks are given in section VI.

\section{Fractional Order Calculus Theory}

Integer order calculus (IOC) has always been the most used tool for representing models, systems and controllers in all applied sciences. Nevertheless, it is required to have a more accurate and close-to-reality representation/modeling of real processes to satisfy more strict requirements, and for doing so, a new calculus tool must be considered [12].

Due to this exigency, and taking as a consideration that not all systems can be generalized to derivatives and integrals of integer order; in the 17th century Leibniz and L'Hôpital raised the question of having a non integer differential order. But it was in the 19th century when eminent scientists like Euler, Laplace, Fourier among others; discussed the possibility of extending the expression (1) for non integers orders " $\alpha$ ". The first real application of the fractional order calculus was made by Abel in 1823, when he discovered 
that the solution for the integral equation for the tautochrone problem could be obtained through an integral in the form of derivative of order $1 / 2$.

$$
{ }_{a} D_{t}^{\alpha}=\left\{\begin{array}{l}
\frac{d^{\alpha}}{d t^{\alpha}}=1 \rightarrow \Re(\alpha)>0 \\
1 \rightarrow \Re(\alpha)=0 \\
\int_{a}^{t}(d \tau)^{-\alpha} \rightarrow \Re(\alpha)<0
\end{array}\right.
$$

It introduced the real order generalization of the integer order calculus, which after allowed to model the voltagecurrent relation of a semi-infinite lossy RC line, or the heat diffusion into a semi-infinite solid as well.

To implement the fractional order calculus, it is required to define an operator that allows non integer differentiation. The most widely known definition for this operator was proposed by Riemann-Liouville [13], which can be appreciated in equation (2) for $(n-1<\alpha<n)$. It can be appreciated that this expression is defined by the convolution operator, which refers to memory phenomena.

$$
{ }_{a} D_{t}^{\alpha}=\frac{1}{\Gamma(n-\alpha)} \frac{d^{n}}{d t^{n}} \int_{a}^{t} \frac{f(\tau)}{(t-\tau)^{\alpha-n+1}}(d \tau),
$$

In this expression, the term $\Gamma(n)$ corresponds to the Euler's gamma function. Even though this function has been widely accepted, the initial conditions have no simple physical interpretation. For this reason, the mathematician Caputo [14] proposed the fractional-order differential operator (see equation 3 ), where initial conditions like $y(0)=y_{0}, \dot{y}(0)=$ $y_{1}$ can be easily interpreted; and $m$ is a natural number immediately superior to $\alpha$, i.e. $(m-1<\alpha<m)$.

$$
{ }_{a} D^{\alpha}=\frac{1}{\Gamma(m-\alpha)} \int_{0}^{t} \frac{f^{(m)}(\tau)}{(t-\tau)^{\alpha-m+1}}(d \tau),
$$

Most of all applied sciences fractional order implementations use the operator introduced by Caputo, due to the practicality of the initial conditions interpretations that it allows. This expression can also be interpreted in the frequency domain through the Laplace or Fourier transform as well, since several applications in system and process engineering are analyzed in this domain.

$$
\mathcal{L}\left\{{ }_{a} D_{t}^{\alpha}\right\}=s^{\alpha} F(s)
$$

In the last decades, the employment of this mathematical tool has grown in the fields of feedback/feed-forward control, signal processing and systems theory; due to the accurate representation of nature process and the flexibility that it provides for designing systems.

\section{A. Fractional Order Controllers Review}

A literature review shows that multiple control techniques are based on integer order calculus, such as ProportionalIntegral-Derivative calculation [9] or Linear Quadratic Regulation [15] among others. They have provided robust solutions to industrial control problems, but the increasing technology and systems complexity, demands more exigent solutions relating to the ones most commonly used. Among the fractional order controllers, it can be found that several implementations have been developed with great results [16], in this section it is presented a brief review of some non integer order control techniques.

1) CRONE: The idea of designing fractional order system to control a dynamic system, was introduced by $\mathrm{A}$. Oustaloup [17] with the CRONE (in french, Commande Robuste d'Ordre Non Entier). For the first generation of the CRONE strategy, its main purpose was to reduce the phase margin variations of the system open loop response.

On the other hand, the main objective of the second generation of the CRONE approach is to directly cancel the phase variations in the gaincross frequency, by ensuring the so-called fractal robustness. To satisfy this requirement, two conditions must be accomplished: a vertical template formed by the open loop Nichols chart, between $-\pi / 2$ and $-\pi$ for the nominal parameters of the plant; and the sliding of the template when the plant is re-parametrized.

The frequency template of the transmittance, i.e.sensitivity function, is given by the function (5), where the $\arg (\beta(j \omega))=-\alpha \pi / 2$ with $1<\alpha<2$, which results in a Nichols chart with a vertical line that crosses the axis in $-\alpha \pi / 2$. This allows the sliding of the system in this straight line when the system parameters vary due to changes in the dynamic behavior, and increases the robustness against plant variations. This fact, explains the main objective of the control design proposed by the CRONE technique, which is to approximate the system open loop response to the frequency template of the transmittance function given by $\beta(\mathrm{s})$.

$$
\beta(s)=\left(\frac{1}{\tau s}\right)^{\alpha}=\left(\frac{\omega_{g c}}{s}\right)^{\alpha}
$$

Finally, assuming that a negative feedback and cascade control architecture is employed, the transfer function for the controller $C(s)$ is obtained from the expression (6), where $G_{p}(s)$ is the plant that is going to be controlled.

$$
C(s)=\frac{\beta(s)}{G_{p}(s)}
$$

Numerous applications of this technique has been carried out, such as car suspension control, hydraulic actuators, among others; bringing great results due to the robustness that this technique provides against model uncertainties or variations.

2) TID Controller: The Tilt-Integral-Derivative Controller [18] proposes a controller that is a modification of the classical Proportional Integral Derivative controller that has provided good results among the years. It has the advantages of the PID controller scheme, but the proportional term is substituted by a tilted compensator whose transfer function is $s^{-\frac{1}{n}}$, (where $n$ is a real number usually between 2 and 3 ) in order to get a response that is reasonably closer to the theoretically optimal Bode response. This compensator is referred as "Tilt", as it provides a feedback gain as a function of frequency which is tilted with respect to the 


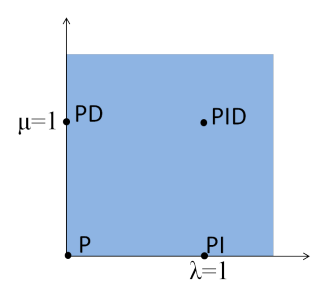

Fig. 1. Complex plane, for fractional order PID controller

gain/frequency of a conventional compensation unit; which is a consequence of having a fractional order integrator. This configuration allows to maintain the system stable under disturbances, even during parameter variations and it can be easily tuned as well.

3) $P I^{\lambda} D^{\mu}$ Controller: The PID controller has been widely used for industrial applications providing good results, and based on that, I. Podlubny [19] proposed to take advantage of the fractional order calculus to get a real order generalization of the PID controller. In the equation (7), it can be seen the transfer function of the controller in the frequency domain.

$$
C(s)=\frac{E(s)}{U(s)}=K p+K d \cdot s^{\mu}+K i \cdot s^{-\lambda} ;(\lambda, \mu)>0
$$

The time domain interpretation of the $P I^{\lambda} D^{\mu}$ controller can be appreciated in the equation (8), where $D$ is the differential operator proposed by Caputo.

$$
u(t)=K_{P} \cdot e(t)+K_{I} \cdot D^{-\lambda} e(t)+K_{D} \cdot D^{\mu} e(t)
$$

The capability of modifying the order of the differential and integral operator, provides a more accurate time and frequency response since it has up to five tuning parameters [20]. The variation of both orders gives as a result the complex plane showed in figure 1 , where by setting both $\lambda$ and $\mu$ as 1 , a classical PID controller is obtained.

The $P I^{\lambda} D^{\mu}$ controller takes advantage of the following features to permit a more accurate frequency response based design:

1) The phase delays or phase variation rate per decade can be varied in the range $[-\pi / 2, \pi / 2]$. For the differential operator, it provides a positive phase increment rate of $\frac{\mu \pi}{4} \mathrm{rad} / \mathrm{sec}$; while the integral operator contributes with a negative phase delay rate of $\frac{\lambda \pi}{4}$ radians per decade.

2) Regarding the magnitude increment/decrement that the differential and integral operator introduce, with this generalization the controller is capable to modify the open loop gain among the range $[-20,20] \mathrm{dB} / \mathrm{dec}$.

These properties bring as a result an increment of the design flexibility as it increases the system robustness. This type of controller rises as a way to achieve more demanding control requirements [21], without losing simplicity and easy-to-tune capabilities. The $P I^{\lambda} D^{\mu}$ controller is one of the most used fractional order controllers in the literature for the good results it has provided [22]; consequently, this is the selected approach that will be further developed in this work.

\section{PROBLEM DESCRIPTION}

As it was previously stated, CACC systems deal with tighter car-following distance by adding V2V communications whereas a string stable response is obtained. A literature review shows different approaches when developing $\mathrm{CACC}$ algorithms, being the most used the robust integer order controllers. For instance, [23] presents a control structure with an integer order PD controller. This proposed structure is used in this paper as a basis for designing the CACC fractional order controller. Before explaining the controller design procedure, the spacing policy that the ego-vehicle will employ to generate the reference inter-distance is described.

\section{A. Spacing Policy}

Different approaches for spacing policies have been proposed in the literature [24], depending on the application upon which it will be implemented e.g. platooning, ACC or CACC. Two main types of spacing policies can be distinguished, the first is the constant spacing policy. This approach has been mostly used for truck or vehicle platoons, where the relative speeds are not considerably high and it is possible to maintain really close inter-distances without any interaction with other lanes. The second type of spacing policy, provides a more flexible conception because it permits to have a variable inter-distance that is function of the headway time. A comparative between this techniques is presented in [25]. The latter approach is selected in this work.

Headway time spacing policy (see figure 2) proposes having a fixed distance $d_{\text {std }}$ added to a variable factor composed by a headway time gap $h_{\text {time }}$ multiplied by the ego-velocity $v(t)$ (see equation 9).

$$
\begin{gathered}
d_{r e f}=d_{s t d}+h_{\text {time }} \cdot v(t) ; h>0 \\
e_{i}=d_{\text {real }}-d_{\text {ref }}
\end{gathered}
$$

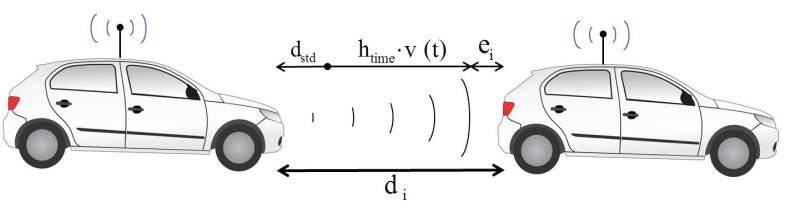

Fig. 2. Representation of a car-following scenario

\section{B. CACC Control Structure}

The main objective of this paper is to propose a novel fractional CACC controller based on fractional calculus. The CACC control structure is adopted from [26] where a feed-forward control algorithm using an integer order PD controller in the feedback loop was proposed. Figure 3 shows a block diagram representation of the CACC system.

For our desing purposes, the vehicle model proposed in [26] was used. According to Fig. 3, $G p(s)$ refers to the 


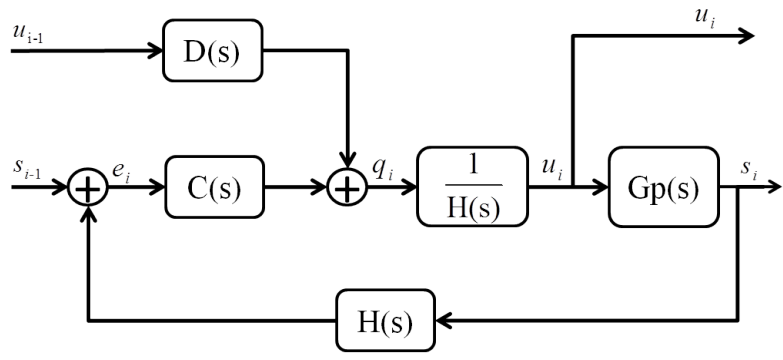

Fig. 3. Control scheme for stability study [26]

vehicle model that can be described by the transfer function (11). It can be appreciated that its output is the vehicle position using an acceleration command as input. Vehicle dynamic is represented by the term $\tau$.

$$
G p(s)=\frac{1}{s^{2}(\tau s+1)}
$$

The transfer function $D(s)$ represents the transport delay due to the communication link between the ego-vehicle and its preceding, and it is defined by the expression $D(s)=$ $e^{-\theta s}$. In the feedback loop, the $H(s)$ expression composes the spacing policy that is applied to obtain the spacing error, which is given to the controller $C(s)$ to generate the control command.

\section{Controller Design}

Fractional controllers can provide better performance whereas keeping simplicity. Using the proposed control architecture, an integer order PD controller provided a robust solution with good results [26]. In spite of this results, one potential problem of the proposed approach is to deal with non-modeled dynamics. The vehicle dynamic plant is built using a simple transfer function that can be oversimplified, specially at low-speeds. Having this in mind, the novel fractional order proportional derivative longitudinal controller (FOPD) is designed paying special attention to increase the system robustness to oversimple vehicle model. This type of controller can benefit from an additional tuning parameter than the classical PD controller. It is the differential operator order which provides the possibility of vary how the controller reacts to the error. Then, the novel FOPD controller contributes to the system relative stability as well as it speeds up the system response to variation in the error incrementing also the robustness against non-modeled dynamics with respect to the integer order PD controllers.

The mathematical expression for this type of controller can be seen in the equation (12), where $K_{p}$ and $K_{d}$ are the gain constants and $\alpha$ is the differentiation order.

$$
C(s)=K_{p}+K_{d} \cdot s^{\alpha} ;\left(K_{p}, K_{d}, \alpha\right)>0
$$

The controller design procedure will be carried out by defining frequency domain requirements. For the sake of clarity, equation (13) can be expressed as:

$$
C(s)=K_{p}+K_{d}\left(\omega^{\alpha} \cos \left(\frac{\alpha \pi}{2}\right)+j \omega^{\alpha} \sin \left(\frac{\alpha \pi}{2}\right)\right)
$$

Controller specifications are defined as follows:

1) Phase margin fulfillment, $\phi_{m} \approx \frac{\pi}{4}$;

$$
\arg \left(G p\left(j \omega_{g c}\right) \cdot C\left(j \omega_{g c}\right)\right)=-\pi+\phi_{m}
$$

2) Open loop gaincross frequency, $\omega_{g c} \approx 1 \mathrm{rad} / \mathrm{sec}$;

$$
\left|G p\left(j \omega_{g c}\right) \cdot C\left(j \omega_{g c}\right)\right|_{d B}=0
$$

3) Robustness to plant gain variations;

$$
\left(\frac{d(\arg (G p(j \omega) \cdot C(j \omega)))}{d \omega}\right)_{\omega=\omega_{g c}}=0
$$

The first two specifications are set to guarantee the controller bandwidth and the phase margin. The third design specification, which aims on having a flat phase in the gaincross frequency, guarantees the good performance to non-modeled dynamics (i.e. model gain changes will not reduce the phase margin). For the first step in the controller design, the model gain and phase (see equation 11) can be described as presented in equations (17) and (18).

$$
\begin{gathered}
|G p(j \omega)|=\frac{1}{\omega^{2} \sqrt{\tau^{2} \omega^{2}+1}} \\
\arg (G p(j \omega))=-\pi+\tan ^{-1}(\tau \omega)
\end{gathered}
$$

On the other side, the controller gain can be determined through the equation (12). In the same line, the controller phase can be as well defined from the expression (13).

$$
\begin{gathered}
|C(j \omega)|=\sqrt{K_{p}^{2}+2 K_{p} K_{d} \cos (\alpha \pi / 2)+K_{d}^{2} \omega^{2 \alpha}} \\
\arg (C(j \omega))=\tan ^{-1}\left(\frac{K_{d} \omega^{\alpha} \sin \left(\frac{\alpha \pi}{2}\right)}{K_{p}+K_{d} \omega^{\alpha} \cos \left(\frac{\alpha \pi}{2}\right)}\right)
\end{gathered}
$$

Therefore, to satisfy the first condition, the equations (18) and (20) are used to assure the phase margin.

$-\tan ^{-1}\left(\tau \omega_{g c}\right)+\tan ^{-1}\left(\frac{K_{d} \omega_{g c}^{\alpha} \sin \left(\frac{\alpha \pi}{2}\right)}{K_{p}+K_{d} \omega_{g c}^{\alpha} \cos \left(\frac{\alpha \pi}{2}\right)}\right)-\phi_{m}=0$

For the second condition, equations (19) and (17) are used to set the gaincross frequency.

$$
\sqrt{K_{p}^{2}+2 K_{p} K_{d} \cos \left(\frac{\alpha \pi}{2}\right)+K_{d}^{2} \omega_{g c}^{2 \alpha}} \cdot \frac{1}{\omega_{g c}^{2} \sqrt{\tau^{2} \omega_{g c}^{2}+1}}=1
$$

For the third specification, that correspond to the phase derivative in $\omega_{g c}$, is analyzed from the controller and model phase in the gaincross frequency.

$$
\frac{K_{p} K_{d} \alpha \sin \left(\frac{\alpha \pi}{2}\right) \omega_{g c}^{\alpha-1}}{K_{p}^{2}+2 K_{p} K_{d} \cos \left(\frac{\alpha \pi}{2}\right) \omega_{g c}^{\alpha}+K_{d}^{2} \omega_{g c}^{2 \alpha}}-\frac{\tau}{1+\tau^{2} \omega_{g c}^{2}}=0
$$




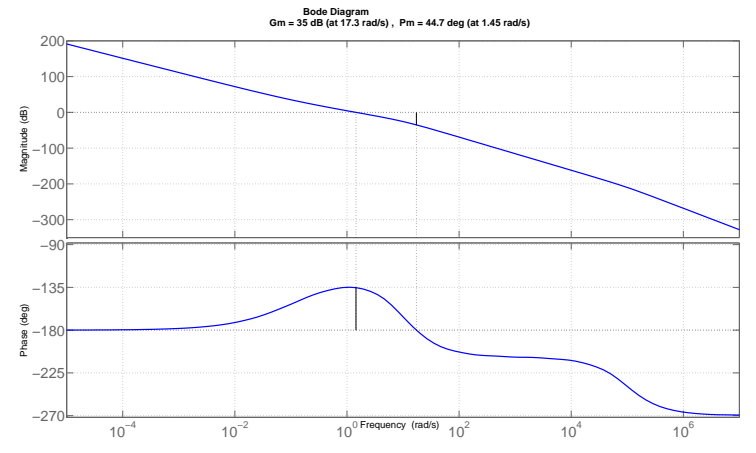

Fig. 4. Bode plot for the open loop response with the parameters obtained

By applying the design procedure described previously, a non-linear equation system is obtained. It can be solved by employing the MATLAB function $f$ solve to find the optimal configuration for $K_{p}, K_{d}$ and $\alpha$ that allows to satisfy the three requirements. Hence, from equations (21), (22) and (23) the controller parameters result: $K p=0.455, K d=$ 1.875 and $\alpha=0.6849$. The open loop response according to this parameter configuration is showed in Fig. 4.

From the Bode plot, it can be appreciated that the phase margin and gaincross frequency specifications are properly satisfied. It can also be seen that the flatness in the phase around the gaincross frequency, assures that the phase margin will be maintained around $-\frac{\pi}{4}$ if non-modeled dynamics are present over the vehicle, that would lead to modifications on the model gain.

\section{RESULTS}

The designed controller was validated using a 6 CACCequipped vehicles string. The leading vehicle followed a speed profile with several speed changes to simulate a real traffic scenario where continuous acceleration/braking maneuvers occur. This profile will allow to assess the whole vehicle string stability response. For the simulation, the V2V communication delays are set at $\theta=0.2 \mathrm{sec}$. The headway time gap applied in the spacing policy is $0.6 \mathrm{sec}$ according to previous studies [27].

Figure 5 shows the vehicle responses to the speed profile using the FOPD CACC controller. The upper plot depicts vehicles' speed. The middle one represents vehicles' acceleration. The bottom plot shows the distance error with respect to the defined spacing policy.

Leader vehicle speed changes were defined within the range of 0 and $22.5 \mathrm{~m} / \mathrm{s}$. For each speed change, a different acceleration command was set to evaluate string response to both aggressive and gentle reference variations. Acceleration changes were limited up to a maximum of $4.5 \mathrm{~m} / \mathrm{s}^{2}$. Finally, the distance error graphically shows whether the string stability is maintained (i.e. the higher the vehicle position, the lower the distance error) or not.

It is important to remark that the leader speed oscillations are accurately tracked by the other vehicles without being amplified downstream, which means that the system is string
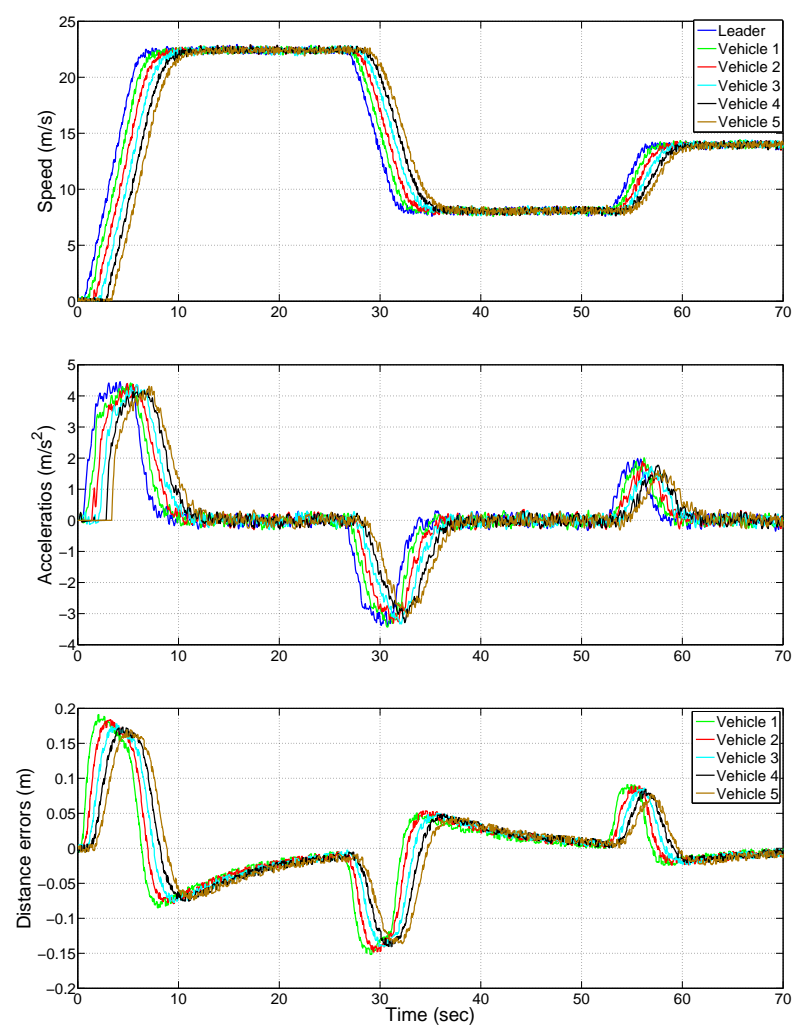

Fig. 5. Performance evaluation for a string of 6 vehicles equipped with CACC

stable. This fact is mostly appreciated in the second and third plot, where the distance errors accelerations are attenuated along the string, for each vehicle. Consequently, the string stability for the developed controller is demonstrated for a headway time gap of $h=0.6 \mathrm{sec}$.

\section{A. String stability analysis}

The system string stability is analyzed by using the control structure showed in Fig. 3. The string stability transfer function can be derived as (24), which must be maintained as less than 1 to guarantee the systems' string stability. By replacing $C(s)$ for the designed fractional controller and keeping the communication delay $\theta=0.2 \mathrm{sec}$, the string stability $(\Gamma(j \omega))$ limit in function of the time gap is studied in fig. 6 .

$$
\Gamma(j \omega)=\frac{D(s)+G_{p}(s) C(s)}{H(s)\left(1+G_{p}(s) C(s)\right)}
$$

One can appreciate how the theoretical minimal time gap to guarantee the string stability is $\mathrm{h}=0.55 \mathrm{sec}$. Then, the string stability is no longer fulfilled, becoming string unstable and, in consequence, amplifying leader speed oscillations.

\section{CONCLUSiOnS}

This paper proposes a novel fractional control algorithms to deal with CACC systems. Firstly, a state-of-the-art review of fractional order calculus is included and a brief comparison with respect to integer order controllers (i.e. PID) 


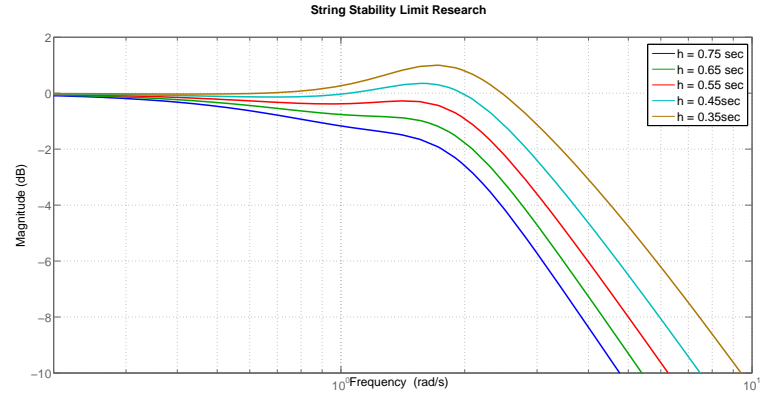

Fig. 6. String stability limit for multiple time gaps, applying the designed controller

is carried out. Then the control architecture, including the time gap policy chosen are detailed. Later, the controller is designed with robustness against non-modeled dynamics that is one of the major problems to deal with when controlling automated vehicles.

The system was tested in simulation using a 6 CACCequipped vehicles string. Vehicles exhibit a good response in terms of not only tracking the gap error regulation but also by keeping string stability. Finally, a string stability analysis to determine the controller boundaries is carried out, being able to provide stable responses up to a time gap equal to $0.55 \mathrm{sec}$.

The future work will be mainly focused on two directions. On one hand, different control structures and configurations will be evaluated to figure out the one that provide better results in terms of stability. Then, real tests on INRIA experimental platforms will be developed to assess controller's performance on real traffic circumstances.

\section{ACKNOWLEDGMENTS}

Authors express their gratitude to the European project AutoNet2030, the french project VALET and the RITS Team for its support in the development of this work.

\section{REFERENCES}

[1] M. Forster, R. Frank, M. Gerla, and T. Engel, "Improving highway traffic through partial velocity synchronization," in Global Cоттиnications Conference (GLOBECOM), 2012 IEEE. IEEE, 2012, pp. 5573-5578.

[2] W. J. Schakel and B. Van Arem, "Improving traffic flow efficiency by in-car advice on lane, speed, and headway," Intelligent Transportation Systems, IEEE Transactions on, vol. 15, no. 4, pp. 1597-1606, 2014.

[3] B. Van Arem, C. J. Van Driel, and R. Visser, "The impact of cooperative adaptive cruise control on traffic-flow characteristics," Intelligent Transportation Systems, IEEE Transactions on, vol. 7, no. 4, pp. 429-436, 2006.

[4] J. Ploeg, A. F. Serrarens, and G. J. Heijenk, "Connect \& drive: design and evaluation of cooperative adaptive cruise control for congestion reduction," Journal of Modern Transportation, vol. 19, no. 3, pp. 207213, 2011.

[5] H. Fritz, A. GERN, H. Schiemenz, and C. Bonnet, "Chauffeur assistant a driver assistance system for commercial vehicles based on fusion of advanced acc and lane keeping," in IEEE intelligent vehicles symposium, 2004, pp. 495-500.

[6] V. Milanés, S. E. Shladover, J. Spring, C. Nowakowski, H. Kawazoe, and M. Nakamura, "Cooperative adaptive cruise control in real traffic situations," Intelligent Transportation Systems, IEEE Transactions on, vol. 15, no. 1, pp. 296-305, 2014.
[7] A. Geiger, M. Lauer, F. Moosmann, B. Ranft, H. Rapp, C. Stiller, and J. Ziegler, "Team annieway's entry to the 2011 grand cooperative driving challenge," Intelligent Transportation Systems, IEEE Transactions on, vol. 13, no. 3, pp. 1008-1017, 2012.

[8] V. Milanés, M. Marouf, J. Perez, D. Gonzalez, and F. Nashashibi, "Low-speed cooperative car-following fuzzy controller for cybernetic transport systems," in Intelligent Transportation Systems (ITSC), 2014 IEEE 17th International Conference on. IEEE, 2014, pp. 2075-2080.

[9] K. J. Åström and T. Hägglund, Advanced PID control. ISA-The Instrumentation, Systems, and Automation Society; Research Triangle Park, NC 27709, 2006.

[10] S. Hassan HosseinNia, I. Tejado, V. Milanés, J. Villagrá, and B. M. Vinagre, "Experimental application of hybrid fractional-order adaptive cruise control at low speed," Control Systems Technology, IEEE Transactions on, vol. 22, no. 6, pp. 2329-2336, 2014

[11] S. H. HosseinNia, I. Tejado, B. M. Vinagre, V. Milanés, and J. Villagrá, "Low speed control of an autonomous vehicle using a hybrid fractional order controller," in Control, Instrumentation and Automation (ICCIA), 2011 2nd International Conference on. IEEE, 2011, pp. 116-121.

[12] C. A. Monje, Y. Chen, B. M. Vinagre, D. Xue, and V. Feliu-Batlle, Fractional-order systems and controls: fundamentals and applications. Springer Science \& Business Media, 2010.

[13] K. Oldham and J. Spanier, "The fractional calculus. 1974," 1974.

[14] M. Caputo, "Linear models of dissipation whose q is almost frequency independent," Annals of Geophysics, vol. 19, no. 4, pp. 383-393, 1966.

[15] P. Shakouri, A. Ordys, D. Laila, and M. Askari, "Adaptive cruise control system: Comparing gain-scheduling pi and lq controllers," $t c$, vol. 2, no. 1, p. 1, 2011.

[16] D. Xue and Y. Chen, "A comparative introduction of four fractional order controllers," in Intelligent Control and Automation, 2002. Proceedings of the 4th World Congress on, vol. 4. IEEE, 2002, pp. 3228-3235.

[17] A. Oustaloup, X. Moreau, and M. Nouillant, "From the second generation crone control to the crone suspension," in Systems, Man and Cybernetics, 1993.'Systems Engineering in the Service of Humans', Conference Proceedings., International Conference on. IEEE, 1993, pp. 143-148.

[18] B. J. Lurie, "Three-parameter tunable tilt-integral-derivative (tid) controller," 1994.

[19] I. Podlubny, "Fractional-order systems and pi/sup/spl lambda//d/sup/spl mu//-controllers," Automatic Control, IEEE Transactions on, vol. 44, no. 1, pp. 208-214, 1999.

[20] C. A. Monje, B. M. Vinagre, V. Feliu, and Y. Chen, "Tuning and auto-tuning of fractional order controllers for industry applications," Control Engineering Practice, vol. 16, no. 7, pp. 798-812, 2008.

[21] B. M. Vinagre and C. A. Monje, "Introducción al control fraccionario," RIAII, vol. 3, no. 3, pp. 5-23, 2006.

[22] I. Podlubny, "Fractional-order systems and fractional-order controllers," Institute of Experimental Physics, Slovak Academy of Sciences, Kosice, vol. 12, 1994.

[23] G. Naus, R. Vugts, J. Ploeg, R. Van De Molengraft, and M. Steinbuch, "Cooperative adaptive cruise control, design and experiments," in American Control Conference (ACC), 2010. IEEE, 2010, pp. 61456150.

[24] D. Swaroop and J. Hedrick, "Constant spacing strategies for platooning in automated highway systems," Journal of dynamic systems, measurement, and control, vol. 121, no. 3, pp. 462-470, 1999.

[25] D. Swaroop and K. Rajagopal, "A review of constant time headway policy for automatic vehicle following," in Intelligent Transportation Systems, 2001. Proceedings. 2001 IEEE. IEEE, 2001, pp. 65-69.

[26] J. Ploeg, B. Scheepers, E. Van Nunen, N. Van de Wouw, and H. Nijmeijer, "Design and experimental evaluation of cooperative adaptive cruise control," in Intelligent Transportation Systems (ITSC), 2011 14th International IEEE Conference on. IEEE, 2011, pp. 260-265.

[27] C. Nowakowski, J. O'Connell, S. E. Shladover, and D. Cody, "Cooperative adaptive cruise control: Driver acceptance of following gap settings less than one second," in Proceedings of the Human Factors and Ergonomics Society Annual Meeting, vol. 54, no. $24 . \quad$ SAGE Publications, 2010, pp. 2033-2037. 